\title{
An Approach to Mathematical Modeling and Estimation of Probe-Drogue Docking Success Probability for UAV Autonomous Aerial Refueling
}

\author{
Xufeng Wang, ${ }^{1,2,3}$ Jianmin $\mathrm{Li}^{2}{ }^{2}$ Xingwei Kong, ${ }^{1}$ Xinmin Dong, ${ }^{1}$ and Bo Zhang ${ }^{2}$ \\ ${ }^{1}$ School of Aeronautics and Astronautics Engineering, Air Force Engineering University, Xian 710038, China \\ ${ }^{2}$ State Key Laboratory of Intelligent Technology and Systems, Tsinghua National Laboratory for Information Science and Technology, \\ Department of Computer Science and Technology, Tsinghua University, Beijing 100084, China \\ ${ }^{3}$ Unit 95835 of PLA, Urumqi 841700, China
}

Correspondence should be addressed to Xufeng Wang; xf-wang14@mails.tsinghua.edu.cn and Xinmin Dong; dongxinmin@139.com

Received 1 March 2017; Revised 8 June 2017; Accepted 2 July 2017; Published 8 August 2017

Academic Editor: Roger L. Davis

Copyright (C) 2017 Xufeng Wang et al. This is an open access article distributed under the Creative Commons Attribution License, which permits unrestricted use, distribution, and reproduction in any medium, provided the original work is properly cited.

\begin{abstract}
One of the keys to the success of aerial refueling for probe-drogue aerial refueling system (PDARS) is the successful docking between the probe and drogue. The study of probe-drogue docking success probability offers an important support to achieving successful docking. During the docking phase of PDARS, based on prior information and reasonable assumptions for the movements of the drogue under atmospheric disturbance, the probe-drogue docking success probability is converted to the probability of the drogue center located in a specific area. A model of the probe-drogue docking success probability is established with and without actuation error, respectively. The curves of the probe-drogue docking success probability with the standard deviation of the drogue central position, the maximum distance from the drogue center position to the equilibrium position, the actuation error, and the standard deviation of the actuation error are obtained through simulations. The study has referential value for the docking maneuver decision of aerial refueling for PDARS.
\end{abstract}

\section{Introduction}

Aerial refueling can greatly improve the ability of manned aircrafts and unmanned aerial vehicles (UAV) to execute remote and long endurance tasks [1-4]. Probe-drogue aerial refueling system (PDARS) has been widely concerned by the research institutions of various countries because of its flexibility and economy [5].

One critical stage of PDARS in the air refueling process is the docking of the probe and drogue [5]. Due to the shaking movement caused by the disturbance during the docking process of PDARS, the PDARS test and assessment are a complex task. The relative position and attitude between the probe and the drogue during the PDARS docking stage was studied in [6-10], and the relative position between the probe and the drogue was obtained accurately by the detection and recognition of the drogue. The hose-drogue movement of the PDARS during the aerial refueling process was analyzed in [11, 12]. In [13], the contacting dynamic behavior model of the probe-drogue during the PDARS docking stage was established, and the collision force and velocity response curve were obtained. The modeling and simulation of hose-drogue shaking phenomenon during the PDARS docking stage were studied in [14-16]. In [17, 18], the dynamic behavior of the hose-drogue whip phenomenon during the PDARS docking stage was modeled, and the influencing factors of the whip phenomenon were analyzed. A detailed study on the docking control during the PDARS aerial refueling process was carried out in $[19,20]$. However, specific research on mathematical modeling and analysis of probe-drogue docking success probability for PDARS has not yet been reported. To the best of our knowledge, only some flight tests show that the docking success probability of the manned aircraft is $30 \% \sim 40 \%$, and the NASA UAV 
aerial refueling docking success probability is about $60 \%$ $[5,17,21]$. As mentioned above, the previous research mainly focused on drogue detection and recognition, drogue $3 \mathrm{D}$ locating, docking control, bow wave effect analysis, and hose whipping control, while it did not involve the probe-drogue docking success probability estimation, which will be of great guiding significance to the decision making of PDARS in the engineering application of probe-drogue aerial refueling [5].

One of the keys to the success of aerial refueling for PDARS is the successful docking between the probe and drogue. The study of probe-drogue docking success probability offers an important support to achieving successful docking. During the process of probe-drogue aerial refueling, the drogue moves faster than the UAV/probe. Therefore, probedrogue aerial refueling can be regarded as the slow dynamic system (namely, the probe) tracking the fast dynamic target (namely, the drogue). In this case, point (namely, the probe) to point (namely, the drogue center) tracking is difficult to dock successfully, but point (namely, the probe) to area (namely, the range of movement of drogue center) tracking can realize successful docking to a certain probability.

Considering the facts that the receiver aircraft operates docking maneuver when the probe is in front of the drogue and the atmospheric disturbance affects the movement of the drogue, therefore, this paper focuses on the theoretical estimation of probe-drogue docking success probability before docking maneuver when the probe is in front of the equilibrium position of drogue center. Firstly, according to the prior information during the docking phase of PDARS, a reasonable assumption for the distribution of the drogue center position under atmospheric disturbance is provided. Then, by converting the probe-drogue docking success probability to the probability of the drogue center located in a specific area, the model of probe-drogue docking success probability is established. The simulation analysis shows the curves of the probe-drogue docking success probability with the standard deviation of the drogue central position, the maximum distance from the drogue central position to the equilibrium position, the actuation error, and the standard deviation of the actuation error, respectively, which can provide an important theoretical basis for the engineering application of PDARS docking maneuver decision during the aerial refueling.

\section{System Design}

We present an approach to mathematical modeling and simulation of probe-drogue docking success probability for UAV autonomous aerial refueling (AAR). Firstly, based on prior information and reasonable assumptions for the movements of the drogue under atmospheric disturbance, the probe-drogue docking success probability is converted to the probability of the drogue center located in a specific area. Then, considering the derivation of the control of UAV, a model of the probe-drogue docking success probability is established with and without actuation error, respectively. Finally, simulations on probe-drogue docking success probability are conducted, and the discussion about considering

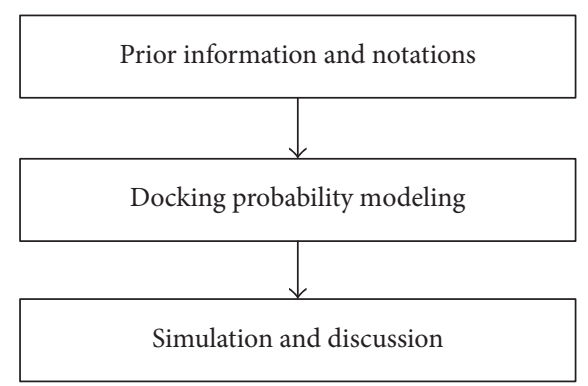

FIGURE 1: Estimation of probe-drogue docking success probability for UAV AAR.

the relative attitude of probe-drogue for docking success probability is given. Figure 1 shows the flowchart of the estimation of probe-drogue docking success probability for UAV AAR.

\section{Prior Information and Notations}

For the simplification of the model of docking success probability, the prior information and notations of probedrogue docking for PDARS are described as follows.

Assumption 1. During a potential successful probe-drogue docking, the drogue randomly meanders in the lateral and vertical displacement by as much as the drogue diameter.

Remark 2. The disturbance, such as atmospheric turbulence and tanker wave, can lead to drogue lateral and vertical displacement $[6,14,22,23]$. A NASA flight test approach to an aerial refueling system indicates that the drogue randomly meanders in the lateral and vertical displacement by as much as the drogue diameter (approximately $61 \mathrm{~cm}$ ), where the atmospheric turbulence is considered, during the docking phase of PDARS [22]. So, considering the reachability and safety of PDARS maneuvers, it is reasonable to assume that, during a potential successful probe-drogue docking, the lateral and vertical displacement of the drogue is less than the drogue diameter, as shown in Figure 2, the red dashed circle. Note that the other direction displacement of the drogue is also less than the drogue diameter, which is a reasonable approximation for estimating the probe-drogue docking success probability for UAV AAR. Thus, the area of disturbed movement of drogue (center) is a circle.

Assumption 3. The lateral and vertical motions of the disturbed drogue are independent.

Remark 4. Considering the shaking movement of the drogue under atmospheric disturbance, the lateral and vertical movements of the drogue have strong independence and weak coupling properties [16]. Therefore, during the docking phase of PDARS, it can be reasonably assumed that the lateral and vertical disturbed drogue motions are independent.

Assumption 5. For specific layout of probe located on the nose of aircrafts, it is reasonable to ignore the bow wave effect. 


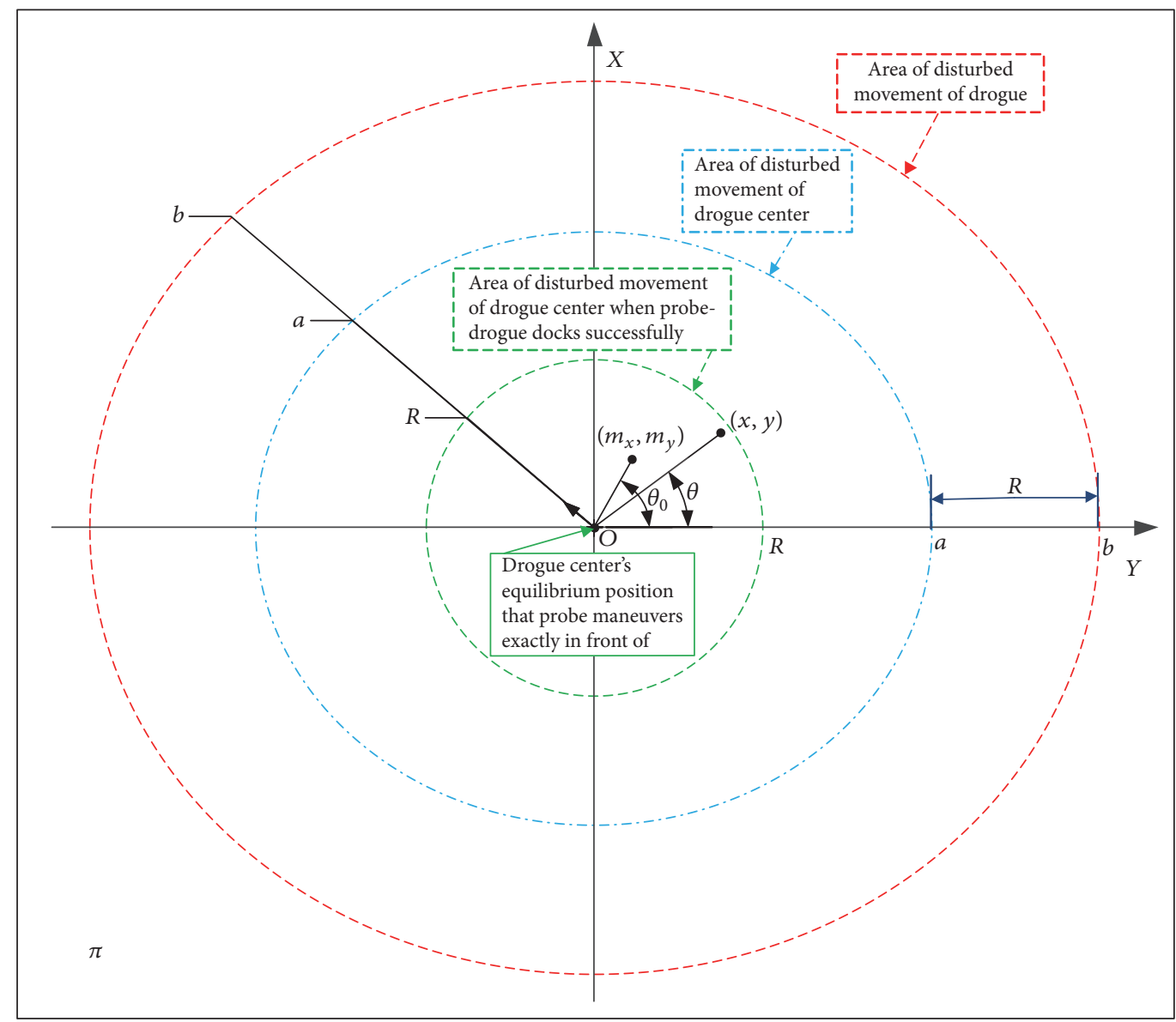

FIGURE 2: Disturbed movement of drogue and docking area without actuation error.

Remark 6. However, in addition to atmospheric turbulence and tanker wake, bow wave also has an effect on the drogue, leading to the drogue leaving the original position when the drogue is close to the receiver aircraft, and this perturbation is obvious for the layout of probe located on the side in manned aircrafts [24-26]. Fortunately, UAV with aerial refueling capability can use the layout of probe located on the nose of aircrafts, which has much less bow wave effect than that of probe located on the side [5]. Therefore, this paper mainly focuses on UAV with the layout of probe located on the nose, without considering bow wave effect. It should be noted that there are references about how to decrease bow wave effect by appropriately locating probe and drogue in practice, such as [27] that proposed a drogue dynamic model under bow wave effect in probe and drogue aerial refueling, which can describe the drogue dynamics during the docking stage and be applicable to a docking controller design to overcome the bow wave effect actively.

Assumption 7. The lateral and vertical components of the drogue center position coordinate are subject to twodimensional normal distribution with isotropic.

Remark 8 . The collected real probe-drogue aerial refueling videos, as shown in http://pan.baidu.com/s/1mi31gQ8, indicate that, during the docking phase of PDARS, frontal images of the drogue can be reasonably expected, and the probe can be approximately perpendicular to the drogue end plane. And in view of the layout of probe located on the nose, this paper mainly focuses on the motion of the drogue in the plane being perpendicular to the forward direction of the UAV. To further clarify the reasonability of the assumption of using a normal distribution to indicate the position of the drogue in the presence of disturbance, the provided real probe-drogue aerial refueling videos, as shown in http://pan.baidu.com/s/1mi31gQ8, can serve as a justification to indicate that assuming a normal distribution is appropriate.

\section{Docking Probability Modeling}

4.1. Model without Actuation Error Considered. According to the above discussion in Section 3, the disturbed movement of drogue and docking area without actuation error are depicted in Figure 2. Note that without actuation error means that the probe maneuvers exactly in front of the drogue equilibrium position, and actuation error $d=\left|O O^{\prime}\right|=\sqrt{x_{c}^{2}+y_{c}^{2}}$ is depicted in Figure 3. As shown in Figure 2, $\pi$ is the drogue end plane, $O$ is the equilibrium position of the drogue center, 


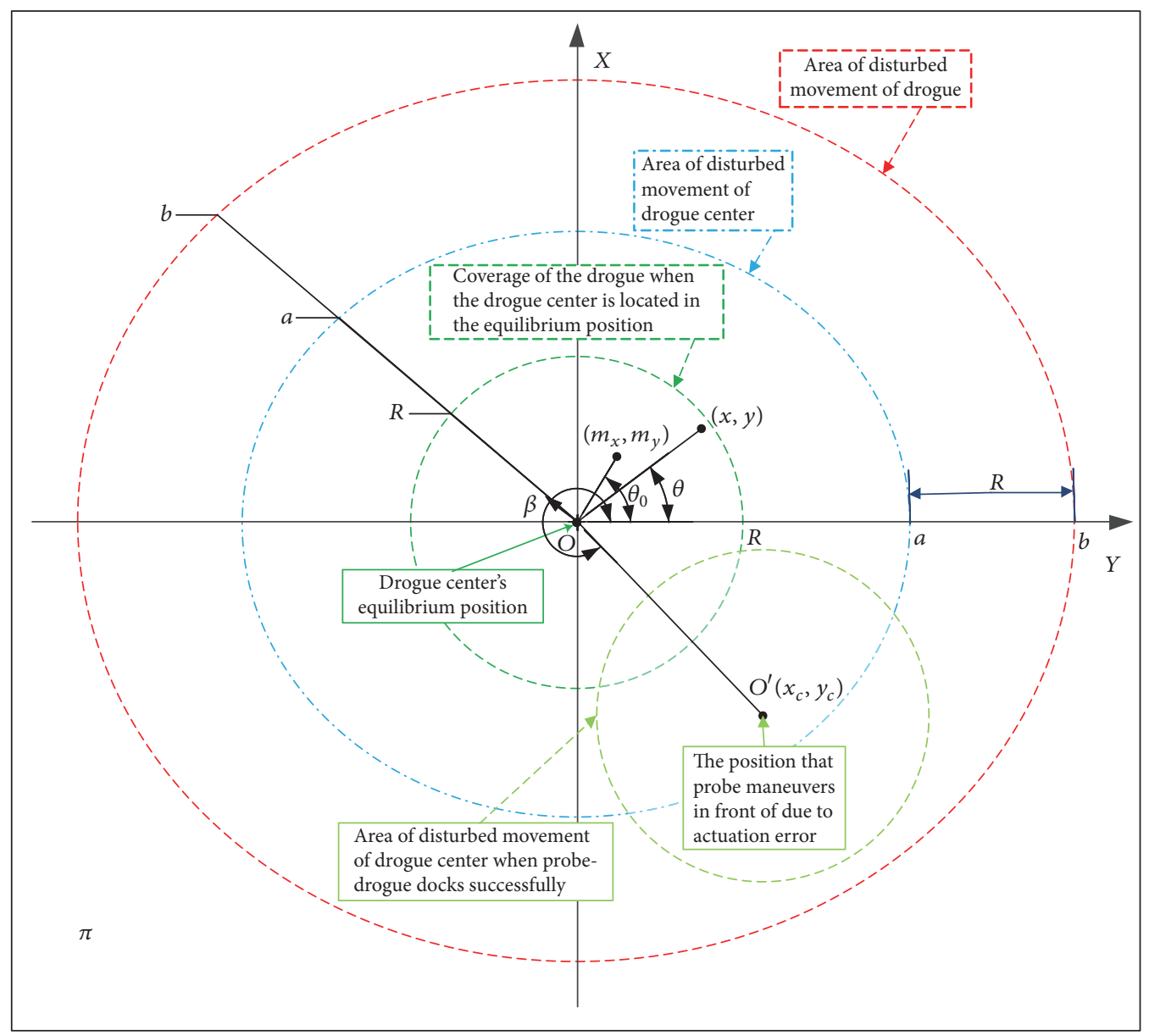

FIGURE 3: Disturbed movement of drogue and docking area with actuation error.

and the radius of the drogue is $R$. When the drogue center is located in the equilibrium position $O$, the coverage of the drogue is $x^{2}+y^{2} \leq R^{2}$. Because during the implementation of refueling docking process, the range of the disturbed movement of the drogue in $X$ and $Y$ direction of the plane $\pi$ is generally not more than the diameter size of the drogue, the perturbed motion of the drogue center is in the range of $x^{2}+y^{2} \leq a^{2}, 0 \leq a \leq 2 R$, and the perturbed motion of the drogue end is in the range of $x^{2}+y^{2} \leq b^{2}, R \leq b=a+R \leq 3 R$, accordingly.

Considering the engineering application of aerial refueling docking, the probe maneuvers gradually right in front of the equilibrium position $O$. Note that the probe maneuvers exactly in front of the equilibrium position $O$, according to corresponding control algorithm, without actuation error means. Therefore, when the drogue center located inside area $x^{2}+y^{2}<R^{2}$, the probe in front of (without actuation error) the equilibrium position $O$ can ensure probe-drogue docking successfully. Namely, the probe-drogue docking success probability is equal to the probability of the drogue center locating in the region $x^{2}+y^{2}<R^{2}$.
The probability density function of the drogue center position $(x, y)$ under disturbance is established as

$$
\begin{aligned}
& f(x, y) \\
& \quad=\frac{1}{2 \pi \sigma_{x} \sigma_{y}} \exp \left\{-\frac{1}{2}\left[\frac{\left(x-m_{x}\right)^{2}}{\sigma_{x}^{2}}+\frac{\left(y-m_{y}\right)^{2}}{\sigma_{y}^{2}}\right]\right\},
\end{aligned}
$$

where $m_{x}$ and $m_{y}$ are the systematic deviation of drogue center in $X$ and $Y$ direction, respectively. $\sigma_{x}^{2}$ and $\sigma_{y}^{2}$ are the variance of the drogue center position in $X$ and $Y$ direction, respectively.

When the polar coordinates are used, there are

$$
\begin{aligned}
x & =r \sin \theta, \\
y & =r \cos \theta, \\
m_{x} & =m \sin \theta_{0}, \\
m_{y} & =m \cos \theta_{0} .
\end{aligned}
$$


The Jacobian determinant of (2) is as follows:

$$
\frac{\partial(x, y)}{\partial(r, \theta)}=\left|\begin{array}{ll}
\frac{\partial x}{\partial r} & \frac{\partial x}{\partial \theta} \\
\frac{\partial y}{\partial r} & \frac{\partial y}{\partial \theta}
\end{array}\right|=\left|\begin{array}{cc}
\sin \theta & r \cos \theta \\
\cos \theta & -r \sin \theta
\end{array}\right|=-r
$$

Therefore, we can obtain the polar coordinate form of $f(x, y)$ defined by

$$
g(r, \theta)=f(x, y)\left|\frac{\partial(x, y)}{\partial(r, \theta)}\right| .
$$

Based on (1)-(5), when $\sigma_{x}=\sigma_{y}=\sigma$, (1) can be transformed into the polar coordinate form

$$
\begin{aligned}
& g(r, \theta)=f(r \sin \theta, r \cos \theta)\left|\frac{\partial(x, y)}{\partial(r, \theta)}\right|=\frac{r}{2 \pi \sigma^{2}} \\
& \cdot \exp \left\{-\frac{1}{2}\left[\frac{\left(r \sin \theta-m \sin \theta_{0}\right)^{2}}{\sigma^{2}}\right.\right. \\
& \left.\left.+\frac{\left(r \cos \theta-m \cos \theta_{0}\right)^{2}}{\sigma^{2}}\right]\right\}=\frac{r}{2 \pi \sigma^{2}} \exp \left\{-\frac{1}{2 \sigma^{2}}\left[r^{2}\right.\right. \\
& \left.\left.+m^{2}-2 r m \cos \left(\theta-\theta_{0}\right)\right]\right\} .
\end{aligned}
$$

To calculate the probe-drogue docking success probability, we then only need to consider the marginal distribution along $r$ (the distance between the drogue center and the equilibrium position $O$ ) as follows:

$$
\begin{aligned}
& p(r)=\int_{0}^{2 \pi} g(r, \theta) d \theta=\frac{r}{\sigma^{2}} \exp \left(-\frac{r^{2}+m^{2}}{2 \sigma^{2}}\right) \int_{0}^{2 \pi} \frac{1}{2 \pi} \\
& \cdot \exp \left[\frac{r m \cos \left(\theta-\theta_{0}\right)}{\sigma^{2}}\right] d \theta=\frac{r}{\sigma^{2}} \exp \left(-\frac{r^{2}+m^{2}}{2 \sigma^{2}}\right) \\
& \cdot I_{0}\left(\frac{r m}{\sigma^{2}}\right),
\end{aligned}
$$

where $I_{0}(\cdot)$ is the first kind zero-order modified Bessel function:

$$
I_{0}(x)=\frac{1}{2 \pi} \int_{0}^{2 \pi} \exp \left[x \cos \left(\theta-\theta_{0}\right)\right] d \theta .
$$

When the observation period is large enough, the systematic deviation of the center position of the drogue in the direction of $X$ and $Y$ can be approximately zero: namely, $m_{x}=$ $0, m_{y}=0$, and $m=0$. Then, we have $I_{0}\left(r m / \sigma^{2}\right)=I_{0}(0)=1$, and therefore, (7) can be simply expressed as

$$
p(r)=\frac{r}{\sigma^{2}} \exp \left(-\frac{r^{2}}{2 \sigma^{2}}\right) .
$$

Then, based on the prior information, when the drogue center located inside area $C_{R}, x^{2}+y^{2}<R^{2}$, the probe in front of (without actuation error) the equilibrium position
$O$ can ensure probe-drogue docking successfully. Namely, the probe-drogue docking success probability is equal to the probability of the drogue center locating in the region $C_{R}$ (with the equilibrium position $O$ as the center and the drogue radius $R$ as the radius):

$$
\begin{aligned}
P & =\iint_{(x, y) \in C_{R}} f(x, y) d x d y=\int_{0}^{R} p(r) d r \\
& =\int_{0}^{R} \frac{r}{\sigma^{2}} \exp \left(-\frac{r^{2}}{2 \sigma^{2}}\right) d r=1-\exp \left(-\frac{R^{2}}{2 \sigma^{2}}\right) .
\end{aligned}
$$

Further, a more intuitive approximate representation between probe-drogue docking success probability and drogue central position is derived. Assuming that, in the observation period $T$ ( $T$ is large enough), a total of $n$ coordinates of drogue center position are observed, namely, $\left(x_{i}, y_{i}\right)$ with $i=1,2, \ldots, n(n>2), \bar{x}=(1 / n) \sum_{i=1}^{n} x_{i}, \bar{y}=$ $(1 / n) \sum_{i=1}^{n} y_{i}$, the drogue equilibrium position $(\bar{x}, \bar{y}) \simeq(0,0)$.

Then, we can obtain the variance of the drogue center position:

$$
\begin{aligned}
\sigma^{2} & =\frac{1}{2}\left[\frac{1}{n-1} \sum_{i=1}^{n}\left(x_{i}-\bar{x}\right)^{2}+\frac{1}{n-1} \sum_{i=1}^{n}\left(y_{i}-\bar{y}\right)^{2}\right] \\
& \simeq \frac{1}{2(n-1)} \sum_{i=1}^{n}\left(x_{i}^{2}+y_{i}^{2}\right)=\frac{1}{2(n-1)} \sum_{i=1}^{n} r_{i}^{2} \\
& \leq \frac{n}{2(n-1)} \max _{i=1,2, \ldots, n}\left\{r_{i}^{2}\right\} .
\end{aligned}
$$

When $n \geq 2$, it follows that

$$
\frac{1}{2} \leq \frac{n}{2(n-1)} \leq 1
$$

and $\lim _{n \rightarrow \infty}(n / 2(n-1))=1 / 2$.

Let

$$
\begin{aligned}
& \max _{i=1,2, \ldots, n}\left\{r_{i}^{2}\right\}=r_{\max }^{2} \\
& \max _{i=1,2, \ldots, n}\left\{r_{i}\right\}=r_{\max }
\end{aligned}
$$

where $r_{\max }$ represents the maximum distance between the drogue center position and the equilibrium position $O$ in the observation period $T$.

Substituting expressions (12), (13) into expression (11) leads to

$$
\sigma^{2} \leq \frac{1}{2} r_{\max }^{2}
$$

Equivalently,

$$
r_{\max \_ \text {inf }}=\sqrt{2} \sigma,
$$

where $r_{\text {max inf }}$ represents the lower-bound value of $r_{\max }$.

Substituting (15) into (10), we get that

$$
P=1-\exp \left(-\frac{R^{2}}{r_{\min }^{2}}\right) .
$$


Because $r_{\max }$ represents the maximum distance between the drogue center position and the equilibrium position $O$ in the observation period $T$. The smaller $r_{\max }$ is, the smaller the disturbed motion of the drogue is, and thus the higher the probe-drogue docking success probability is. Therefore, statistically, the probe-drogue docking success probability satisfies the following relation:

$$
\begin{aligned}
P\left(r_{\text {max_sup }}=\xi\right) & =P\left(r_{\max } \leq \xi\right) \geq P\left(r_{\max } \geq \xi\right) \\
& =P\left(r_{\text {max_inf }}=\xi\right),
\end{aligned}
$$

where $r_{\text {max_sup }}$ represents the upper-bound value of $r_{\text {max }}$, with $\xi$ being the threshold.

The proof of expression (17) is as follows.

Proof. There exists $r_{\text {max_sup }}=\xi \Leftrightarrow r_{\max } \leq \xi$ and $r_{\text {max_inf }}=$ $\xi \Leftrightarrow r_{\max } \geq \xi$, such that

$$
\begin{gathered}
P\left(r_{\text {max _sup }}\right)=P\left(r_{\max } \leq \xi\right), \\
P\left(r_{\max } \geq \xi\right)=P\left(r_{\text {max_inf }}=\xi\right) .
\end{gathered}
$$

Moreover, because the docking success probability when $r_{\max } \leq \xi$ is bound to be no less than the docking success probability when $r_{\max } \geq \xi$, it can be deduced that

$$
P\left(r_{\max } \leq \xi\right) \geq P\left(r_{\max } \geq \xi\right) .
$$

Thus, by expressions (18) and (19), expression (17) is established.

The proof is completed.

Note that the probability estimation based on $r_{\max }$ is more intuitive and conservative, while the probability estimation based on $\sigma$ is more comprehensive and accurate. Thus, the two estimation methods have their own advantages and the docking success probability should be estimated based on the two methods simultaneously.

4.2. Model with Actuation Error Considered. Under the condition with actuation error, because of the existence of the actuation error, the docking position of the probedrogue deviates from $O(0,0)$, the equilibrium position of the movement of drogue center. The disturbed movements of drogue and docking area with actuation error are depicted in Figure 3. As shown in Figure 3, $O^{\prime}\left(x_{c}, y_{c}\right)$ is the docking position, the actuation error $d=\left|O O^{\prime}\right|=\sqrt{x_{c}^{2}+y_{c}^{2}}, \beta$ is the angle between $d$ and $y$ axis, $x_{c}=d \sin \beta$, and $y_{c}=$ $d \cos \beta$. Thus, under the condition with actuation error, in order to make the probe-drogue docking successfully, the drogue center must be located within the region $\left(x-x_{c}\right)^{2}+$ $\left(y-y_{c}\right)^{2} \leq R^{2}$. Therefore, under the condition with actuation error, the probe-drogue docking success probability is equal to the probability that the drogue center is located in the region $\left(x-x_{c}\right)^{2}+\left(y-y_{c}\right)^{2} \leq R^{2}$.

As mentioned above, under the condition with actuation error, the probe-drogue docking success probability is equal to the probability of the drogue center locating in the region
$C_{R}^{\prime}:\left(x-x_{c}\right)^{2}+\left(y-y_{c}\right)^{2}<R^{2}$ (with $O^{\prime}\left(x_{c}, y_{c}\right)$ as the center and the drogue radius $R$ as the radius):

$$
\begin{aligned}
& P=\iint_{(x, y) \in C_{R}^{\prime}} f(x, y) d x d y \\
& =\iint_{\left(x-x_{c}\right)^{2}+\left(y-y_{c}\right)^{2} \leq R^{2}} \frac{1}{2 \pi \sigma^{2}} \exp \left(-\frac{x^{2}+y^{2}}{2 \sigma^{2}}\right) d x d y \\
& =\iint_{(x-d \sin \beta)^{2}+(y-d \cos \beta)^{2} \leq R^{2}} \frac{1}{2 \pi \sigma^{2}} \exp \left(-\frac{x^{2}+y^{2}}{2 \sigma^{2}}\right) d x d y \\
& =\iint_{x^{2}+(y-d)^{2} \leq R^{2}} \frac{1}{2 \pi \sigma^{2}} \exp \left(-\frac{x^{2}+y^{2}}{2 \sigma^{2}}\right) d x d y \\
& =\iint_{(x-d)^{2}+y^{2} \leq R^{2}} \frac{1}{2 \pi \sigma^{2}} \exp \left(-\frac{x^{2}+y^{2}}{2 \sigma^{2}}\right) d x d y \\
& =P_{\sigma, d}(\sigma, d),
\end{aligned}
$$

where $\sigma^{2}$ is the variance of the drogue center position in $X$ and $Y$ direction. And because $R$ is a constant, (20) shows that the probe-drogue docking success probability $P_{\sigma, d}(\sigma, d)$ is a function of $\sigma$ and $d$, under the condition with actuation error.

Further, from the view of statistics, the relationship between the probability of successful docking and the variance of the control deviation is derived.

Under the condition with actuation error, given that the variance of the control deviation $d$ in $X$ and $Y$ direction is $\sigma_{c}^{2}$. Now we define $P_{\sigma, \sigma_{c}}\left(\sigma, \sigma_{c}\right)$ as the probe-drogue docking success probability, a function of $\sigma$ and $\sigma_{c}$, under the condition with actuation error. Next, the relationship between $P_{\sigma, \sigma_{c}}\left(\sigma, \sigma_{c}\right)$ and $P_{\sigma, d}(\sigma, d)$ is derived, and the relationship of the probe-drogue docking success probability versus $\sigma$ and $\sigma_{c}$ is given. The derivation process of the relationship between $P_{\sigma, \sigma_{c}}\left(\sigma, \sigma_{c}\right)$ and $P_{\sigma, d}(\sigma, d)$ is as follows.

According to expression (14), we can obtain

$$
\sigma_{c} \leq \frac{1}{\sqrt{2}} d_{\max }
$$

where $d_{\max }$ represents the maximum of the actuation error in the observation period $T$.

Due to the smaller bias of the control, the higher probability of probe-drogue docking success, we have

$$
\begin{aligned}
P_{\sigma, \sigma_{c}}\left(\sigma, \sigma_{c}\right) & =P_{\sigma, \sigma_{c}}\left(\sigma, \sigma_{c} \leq \frac{1}{\sqrt{2}} d_{\max }\right) \\
& \geq P_{\sigma, \sigma_{c}}\left(\sigma, \sigma_{c}=\frac{1}{\sqrt{2}} d_{\max }\right) .
\end{aligned}
$$

It can be proved that

$$
P_{\sigma, \sigma_{c}}\left(\sigma, \sigma_{c}=\frac{1}{\sqrt{2}} d_{\max }\right) \geq P_{\sigma, d}\left(\sigma, d=\sqrt{2} \sigma_{c}\right) \text {. }
$$

From the intuitive understanding, expression (23) was established because the docking success probability when $d \leq$ $\sqrt{2} \sigma_{c}$ (namely, $\left.P_{\sigma, \sigma_{c}}\left(\sigma, \sigma_{c}=(1 / \sqrt{2}) d_{\max }\right)\right)$ is bound to be no less than the docking success probability when $d=\sqrt{2} \sigma_{c}$ (namely, $P_{\sigma, d}\left(\sigma, d=\sqrt{2} \sigma_{c}\right)$ ).

The proof of expression (23) is as follows. 
Proof. Let $\sigma_{c}=(1 / \sqrt{2}) d_{\max }$. Then there exists $d=d_{1} \leq$ $d_{\max }=\sqrt{2} \sigma_{c}$ such that

$$
\begin{aligned}
& P_{\sigma, \sigma_{c}}\left(\sigma, \sigma_{c}=\frac{1}{\sqrt{2}} d_{\max }\right) \\
& \quad=P_{\sigma, d}\left(\sigma, d=d_{1} \leq d_{\max }=\sqrt{2} \sigma_{c}\right) .
\end{aligned}
$$

Moreover, it can be deduced that

$$
\begin{array}{r}
P_{\sigma, d}\left(\sigma, d=d_{1} \leq d_{\max }=\sqrt{2} \sigma_{c}\right) \\
\geq P_{\sigma, d}\left(\sigma, d=d_{\max }=\sqrt{2} \sigma_{c}\right) .
\end{array}
$$

Thus, by expressions (24) and (25), expression (23) is established.

The proof is completed.

Then, from expressions (22) and (23), we get that

$$
P_{\sigma, \sigma_{c}}\left(\sigma, \sigma_{c}\right) \geq P_{\sigma, d}\left(\sigma, d=\sqrt{2} \sigma_{c}\right)
$$

Expression (26) is the relationship between $P_{\sigma, \sigma_{c}}\left(\sigma, \sigma_{c}\right)$ and $P_{\sigma, d}(\sigma, d)$. Namely,

$$
P_{\sigma, \sigma_{c}}\left(\sigma, \sigma_{c}\right) \geq P_{\sigma, \sigma_{c}}\left(\sigma, \sigma_{c}\right)_{\mathrm{inf}},
$$

where $P_{\sigma, \sigma_{c}}\left(\sigma, \sigma_{c}\right)_{\text {inf }}$ represents the lower-bound value of $P_{\sigma, \sigma_{c}}\left(\sigma, \sigma_{c}\right)$ and

$$
\begin{aligned}
& P_{\sigma, \sigma_{c}}\left(\sigma, \sigma_{c}\right)_{\mathrm{inf}}=P_{\sigma, d}\left(\sigma, d=\sqrt{2} \sigma_{c}\right) \\
& =\iint_{\left(x-\sqrt{2} \sigma_{c} \sin \beta\right)^{2}+\left(y-\sqrt{2} \sigma_{c} \cos \beta\right)^{2} \leq R^{2}} \frac{\exp \left(-\left(x^{2}+y^{2}\right) / 2 \sigma^{2}\right) d x d y}{2 \pi \sigma^{2}} \\
& =\iint_{x^{2}+\left(y-\sqrt{2} \sigma_{c}\right)^{2} \leq R^{2}} \frac{1}{2 \pi \sigma^{2}} \exp \left(-\frac{x^{2}+y^{2}}{2 \sigma^{2}}\right) d x d y \\
& =\iint_{\left(x-\sqrt{2} \sigma_{c}\right)^{2}+y^{2} \leq R^{2}} \frac{1}{2 \pi \sigma^{2}} \exp \left(-\frac{x^{2}+y^{2}}{2 \sigma^{2}}\right) d x d y .
\end{aligned}
$$

\section{Simulations and Analyses}

According to $[14,22]$, we set the drogue diameter $D$ to be $0.61 \mathrm{~m}$, and, namely, the drogue radius $R$ is $0.305 \mathrm{~m}$.

5.1. Without Actuation Error Considered. Based on (10), the probe-drogue docking success probability $P$ versus $\sigma$ is shown in Figures 4 and 5.

Based on (16), the probe-drogue docking success probability $P$ versus $r_{\text {max_inf }}$ is depicted in Figures 6 and 7 . Note that $P\left(r_{\text {max_sup }}=\xi\right)=P\left(r_{\max } \leq \xi\right) \geq P\left(r_{\text {max inf }}=\xi\right)$.

The variation ratio of the probe-drogue docking success probability $P$ versus $\sigma$ is depicted in Figure 8.

The variation ratio of the probe-drogue docking success probability $P$ versus $r_{\text {max_inf }}$ is depicted in Figure 9.

Through the analysis of Figures 4-9, we can get the following:

(1) During the probe-drogue docking process, the more stable the drogue central position, the larger the probe-drogue docking success probability.
(2) The probe-drogue docking success probability drops from $89.46 \%$ to $63.21 \%$ rapidly when $r_{\text {max_inf }}$ increases from $20 \mathrm{~cm}$ to $30 \mathrm{~cm}$, which provides a docking maneuver threshold to some extent. Because that aerial refueling is a high difficult task requiring stability, accuracy, and safety, to ensure the success of the aerial refueling, the value of $r_{\text {max sup }}$ can be used as a decision reference of probe-drogue docking maneuver. When $r_{\text {max_sup }}=R$, the probe-drogue docking success probability $P\left(r_{\text {max sup }}=R\right) \geq$ $63.21 \%$. When $r_{\text {max_sup }}=(2 / 3) R$, the probe-drogue docking success probability $P\left(r_{\text {max_sup }}=(2 / 3) R\right) \geq$ $89.46 \%$. When $r_{\text {max_sup }}=(1 / 2) R$, the probe-drogue docking success probability $P\left(r_{\text {max sup }}=(1 / 2) R\right) \geq$ 98.17\%. Therefore, the docking maneuver should be made when $r_{\text {max_sup }}<(2 / 3) R \approx 20 \mathrm{~cm}$ for safety. Note that the decision threshold $r_{\text {max_sup }}=\xi$ (namely, $\left.r_{\max } \leq \xi\right)$ of the probe-drogue docking maneuver during the aerial refueling deserves further extensive flight tests and comprehensive assessments to determine a reasonable decision threshold.

(3) For the probe-drogue docking success probability/rate, a comparison between the theoretical analysis proposed in this paper and the flight test results of NASA AAR demonstration [21] is described as follows, with a detailed introduction of the NASA results and a visualized comparison in Figure 10. Note that the probe-drogue docking success probability is corresponding to the theoretical analysis proposed in this paper, and the probe-drogue docking success rate is corresponding to the flight test results of NASA AAR demonstration [21].

Figure 10 shows the visualized comparison between the flight test results of NASA AAR demonstration [21] and the theoretical analysis proposed in this paper for the probedrogue docking success rate/probability. Figure 10(a) represents the left view of the drogue and $R_{C}$ value, corresponding to a 90 percent probe-drogue docking success rate in the flight test of NASA AAR demonstration [21]. Figure 10(b) describes the main view of the drogue and $r_{\text {max_sup }}$ value, corresponding to larger than 89.44 percent probe-drogue docking success probability in the theoretical analysis proposed in this paper.

As shown in Figure 10(a), according to the flight test results of NASA AAR demonstration [21], $R_{C}$ is the capture radius that was defined as being $10.16 \mathrm{~cm}$ inside the outer ring of the drogue, which was suggested by the project pilot as a diameter that would result in a 90 percent probedrogue docking success rate with minimal vertical and lateral velocity. Actually, $R_{C}$ defines a tube coaxial (obtained by revolving the blue area $180^{\circ}$ about the blue dash dot line) to the drogue, and when the probe is located within the capture radius $R_{C}$ of the drogue, the probe-drogue docking success rate would reach 90 percent [21].

As shown in Figure 10(b), according to the theoretical analysis proposed in this paper, when $r_{\text {max_sup }}=20.34 \mathrm{~cm}$, which means the probe is located within the capture radius $20.34 \mathrm{~cm}$ of the drogue in practical engineering (namely, $r_{\text {max sup }}=20.34 \mathrm{~cm}$ is equal to $R_{C}=20.34 \mathrm{~cm}$ in practical 


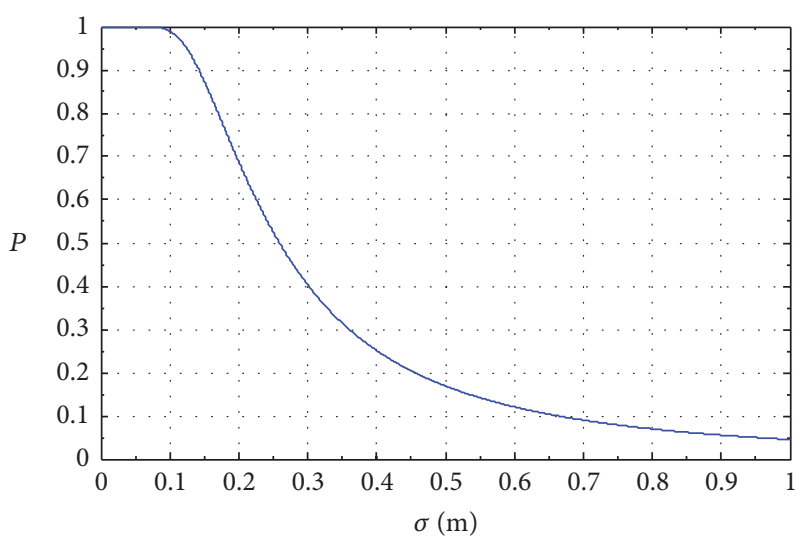

FIgURE 4: Probe-drogue docking success probability $P$ versus $\sigma$.

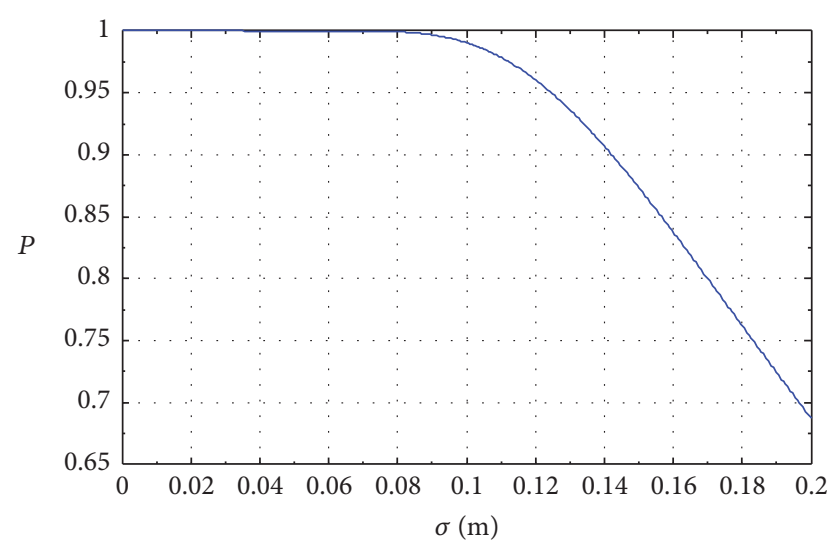

Figure 5: $P$ versus $\sigma$ (local magnification).

engineering), the probe-drogue docking success probability $P\left(r_{\text {max_sup }}=0.2034 \mathrm{~m}\right) \geq 89.44 \%$.

From the above discussion, it can be inferred that " $R_{C}$ $=20.34 \mathrm{~cm}$ in Figure 10(a)" is equal to " $r_{\text {max_sup }}=20.34 \mathrm{~cm}$ in Figure 10(b)" in practical engineering, which result in 90 percent probe-drogue docking success rate in the flight test results of NASA AAR demonstration [21] and 89.44 percent probe-drogue docking success probability in the theoretical analysis proposed in this paper, respectively. Therefore, for the probe-drogue docking success probability/rate, the theoretical analysis proposed in this paper is consistent with the flight test results of NASA AAR demonstration [21].

5.2. With Actuation Error Considered. Under the condition with actuation error, based on (20), the probe-drogue docking success probability $P_{\sigma, d}(\sigma, d)$ versus $\sigma$ and $d$ is shown in Figure 11.

From Figure 11, the following is shown:

(1) Under the condition with actuation error, when $0 \leq$ $d<R=0.305 \mathrm{~m}$, the probe-drogue docking success probability $P_{\sigma, d}(\sigma, d)$ decreases with the increase of $\sigma$ and $d$. The larger the actuation error $d$ is, the faster the probe-drogue docking success probability $P_{\sigma, d}(\sigma, d)$ decreases with the increase of $\sigma$.

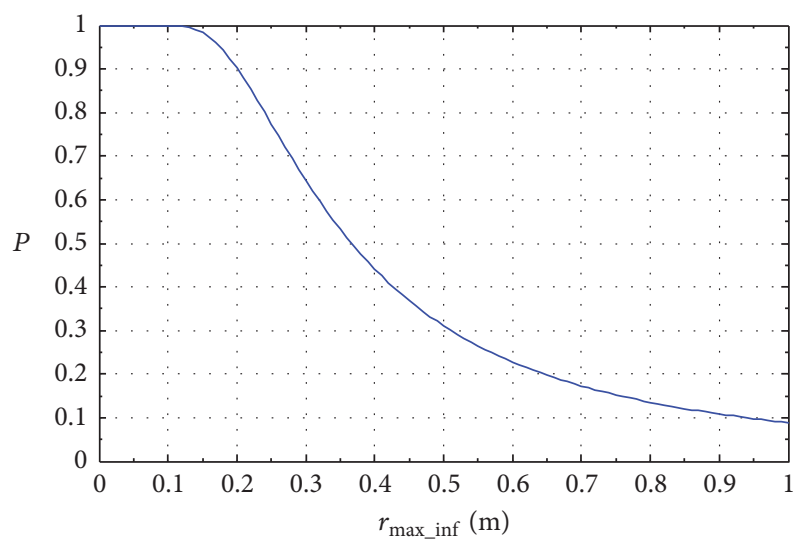

Figure 6: $P$ versus $r_{\text {max _inf }}$.

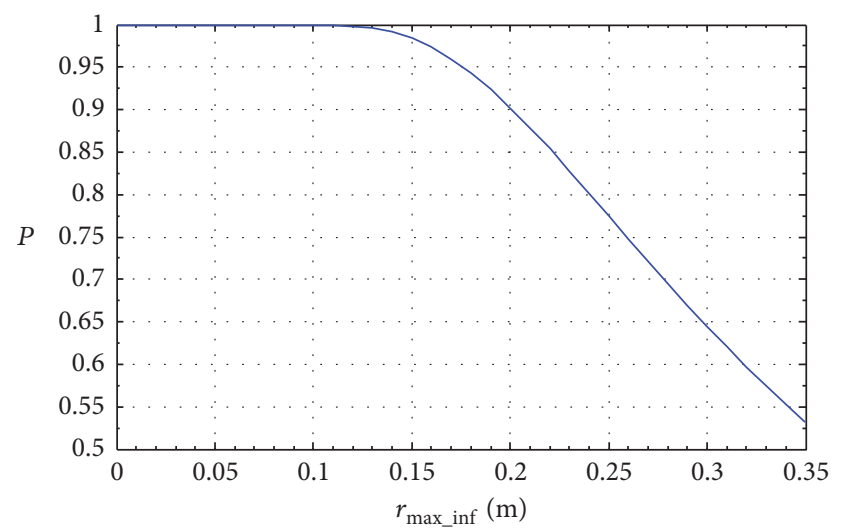

Figure 7: $P$ versus $r_{\text {max_inf }}$ (local magnification).

(2) Under the condition with actuation error, $d=R=$ $0.305 \mathrm{~m}$ is the separatrix. When $d>R=0.305 \mathrm{~m}$, the probe-drogue docking success probability $P_{\sigma, d}(\sigma, d)$ remains at a low level with $P_{\sigma, d}(\sigma, d)_{\max }<0.5$.

(3) Because of the accuracy and safety requirements of the aerial refueling mission, the study of $P_{\sigma, d}(\sigma, d)$ with $0 \leq d<R=0.305 \mathrm{~m}$ will be more meaningful and helpful. Considering that when $0 \leq d<R=$ $0.305 \mathrm{~m}$, the high value of $P_{\sigma, d}(\sigma, d)$ is basically in the range of $0 \leq \sigma \leq 0.2 \mathrm{~m}$. In view of this, we choose the range of $0 \leq d<R=0.305 \mathrm{~m}$ with $0 \leq \sigma \leq 0.2 \mathrm{~m}$ and further give the probe-drogue docking success probability $P_{\sigma, d}(\sigma, d)$ versus $\sigma$ and $d$ in local detail, as shown in Figure 12.

From Figure 12, it can be seen in more detail that, in $P_{\sigma, d}(\sigma, d)$ high value range $(0 \leq d<R=0.305 \mathrm{~m}$ with $0 \leq \sigma \leq 0.2 \mathrm{~m})$, the existence of actuation error $d$ can have a negative impact on the $P_{\sigma, d}(\sigma, d)$. When $\sigma$ is certain (namely, under the same external environment), the greater the actuation error $d$, the larger the decline of $P_{\sigma, d}(\sigma, d)$. That is, the greater the actuation error $d$, the higher requirements of the external environment when making docking maneuver for UAV AAR. Consequently, it is necessary to take appropriate control strategy to reduce the 


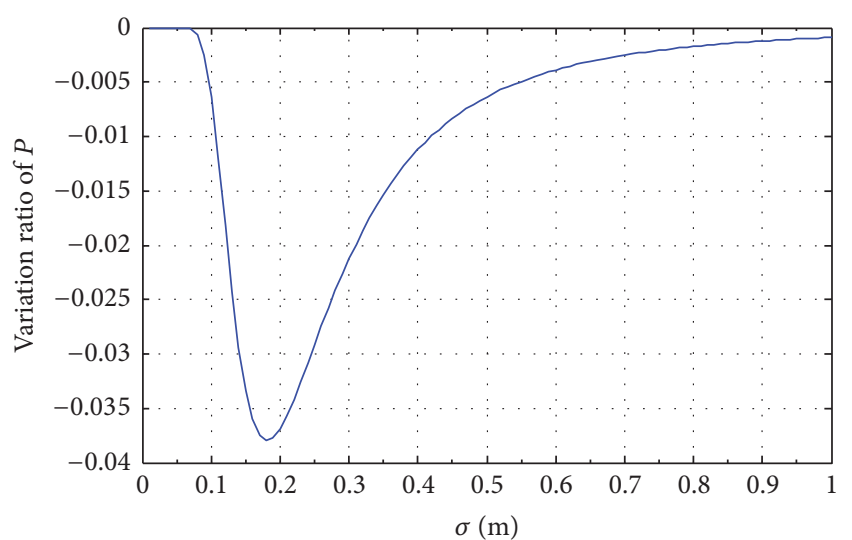

Figure 8: Variation ratio of $P$ versus $\sigma$.

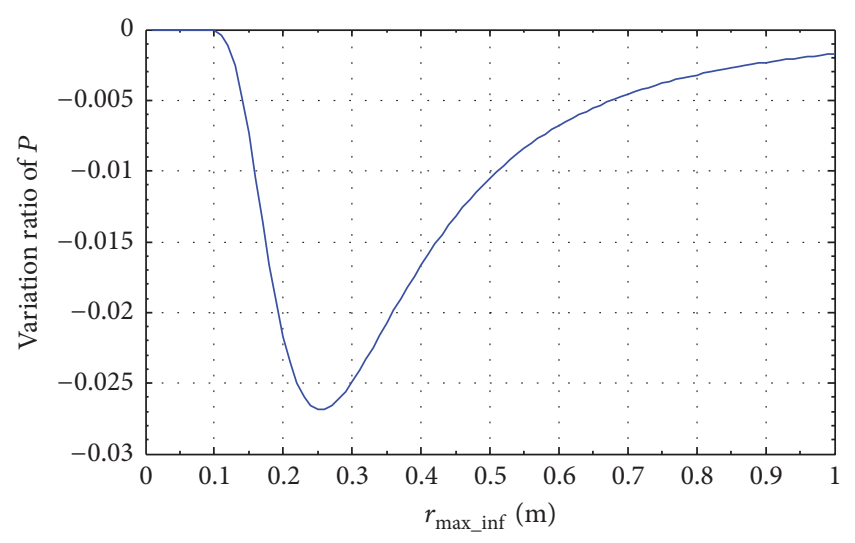

Figure 9: Variation ratio of $P$ versus $r_{\text {max_inf }}$.

actuation error $d$, and when actuation error $d$ is small, we can ensure high probe-drogue docking success probability even when the external disturbance is relatively large, which increases the adaptability to the external environment of AAR.

Under the condition with actuation error, based on (28), the lower-bound value of probe-drogue docking success probability $P_{\sigma, \sigma_{c}}\left(\sigma, \sigma_{c}\right)_{\text {inf }}$ versus $\sigma$ and $\sigma_{c}$ is shown in Figure 13. Note that the probe-drogue docking success probability $P_{\sigma, \sigma_{c}}\left(\sigma, \sigma_{c}\right) \geq P_{\sigma, \sigma_{c}}\left(\sigma, \sigma_{c}\right)_{\text {inf }}$.

From Figure 13, the following is shown:

(1) Under the condition with actuation error, when $0 \leq$ $\sigma_{c}<0.2157 \mathrm{~m}$, the lower-bound value of probedrogue docking success probability $P_{\sigma, \sigma_{c}}\left(\sigma, \sigma_{c}\right)_{\text {inf }}$ decreases with the increase of $\sigma$ and $\sigma_{c}$. The larger $\sigma_{c}$ is, the faster $P_{\sigma, \sigma_{c}}\left(\sigma, \sigma_{c}\right)_{\text {inf }}$ decreases with the increase of $\sigma$.

(2) Under the condition with actuation error, $\sigma_{c}=$ $0.2157 \mathrm{~m}$ is the separatrix. When $\sigma_{c}>0.2157 \mathrm{~m}$, $P_{\sigma, \sigma_{c}}\left(\sigma, \sigma_{c}\right)_{\text {inf }}$ remains at a low level with $P_{\sigma, \sigma_{c}}(\sigma$, $\left.\sigma_{c}\right)_{\text {inf }}<0.5$.

(3) Because of the accuracy and safety requirements of the aerial refueling mission, the study of $P_{\sigma, \sigma_{c}}\left(\sigma, \sigma_{c}\right)_{\text {inf }}$ with $0 \leq \sigma_{c}<0.2157 \mathrm{~m}$ will be more meaningful and helpful. Consider that when $0 \leq \sigma_{c}<0.2157 \mathrm{~m}$, the high value of $P_{\sigma, \sigma_{c}}\left(\sigma, \sigma_{c}\right)_{\text {inf }}$ is basically in the range of $0 \leq \sigma \leq 0.2 \mathrm{~m}$. In view of this, we choose the range of $0 \leq \sigma_{c}<0.2157 \mathrm{~m}$ with $0 \leq \sigma \leq 0.2 \mathrm{~m}$ and further give $P_{\sigma, \sigma_{c}}\left(\sigma, \sigma_{c}\right)_{\text {inf }}$ versus $\sigma$ and $\sigma_{c}$ in local detail, as shown in Figure 14. Note that the probedrogue docking success probability $P_{\sigma, \sigma_{c}}\left(\sigma, \sigma_{c}\right) \geq$ $P_{\sigma, \sigma_{c}}\left(\sigma, \sigma_{c}\right)_{\text {inf }}$.

From Figure 14, it can be seen in more detail that, in $P_{\sigma, \sigma_{c}}\left(\sigma, \sigma_{c}\right)_{\text {inf }}$ high value range $\left(0 \leq \sigma_{c}<0.2157 \mathrm{~m}\right.$ with $0 \leq \sigma \leq 0.2 \mathrm{~m}$ ), the existence of $\sigma_{c}$ can have a negative impact on $P_{\sigma, \sigma_{c}}\left(\sigma, \sigma_{c}\right)_{\text {inf }}$. When $\sigma$ value is certain (namely, under the same external environment), the greater $\sigma_{c}$, the larger decline of $P_{\sigma, \sigma_{c}}\left(\sigma, \sigma_{c}\right)_{\text {inf }}$. That is, the greater $\sigma_{c}$, the higher requirements of the external environment when making docking maneuver for UAV AAR. Consequently, it is necessary to take appropriate control strategy to reduce $\sigma_{c}$, and when $\sigma_{c}$ is small, we can ensure high probe-drogue docking success probability even when the external disturbance is relatively large, which increases the adaptability to the external environment of AAR.

\section{Discussions}

6.1. Considering the Relative Attitude of Probe-Drogue for Docking Success Probability. Note that indeed the movement of drogue center has an important effect on the success for docking, but there are many other factors that can produce effect on the success of docking. In order to make the proposed model of probe-drogue docking success probability more comprehensive and reasonable, we further consider the relative attitude between the drogue and the probe, which is an important factor for a successful docking. Namely, a more reasonable probe-drogue docking success probability P_optimal can be estimated, according to the relative attitude between the drogue and the probe, based on the initial probe-drogue docking success probability $P$ initial using the movement mathematical model of drogue center. Details are as follows.

Due to the rolling symmetry property of drogue, we only consider its pitch angle $\theta$ and yaw angle $\varphi$. Note that the theory, experiment, and precision of drogue attitude measurement have been described in our previous work [6] in detail. So, in this study, we assume that accurate parameters, such as the pitch angle $\theta$ and yaw angle $\varphi$ of the drogue, can be obtained precisely.

During the process of aerial refueling, there is a setting that when the probe is right in front of the drogue and the probe is perpendicular to the end of the drogue, then the pitch angle $\theta$ and yaw angle $\varphi$ of the drogue are all $0^{\circ}$. Then, theoretically, there exist $\theta \in[-\pi, \pi]$ and $\varphi \in[-\pi, \pi]$. We should note that, during the docking phase of aerial refueling, $30^{\circ}$ deflection of the drogue can be regarded as large, and when the deflection is more than $90^{\circ}$, the probe and drogue cannot dock successfully.

When $\theta=0^{\circ}$ and $\varphi=0^{\circ}, P \_o p t i m a l$ is equal to $P$ initial; when the deflection of the drogue increases from $0^{\circ}$ to $90^{\circ}$, $P \_o p t i m a l$ decreases from $P$ initial to 0 ; when the deflection 


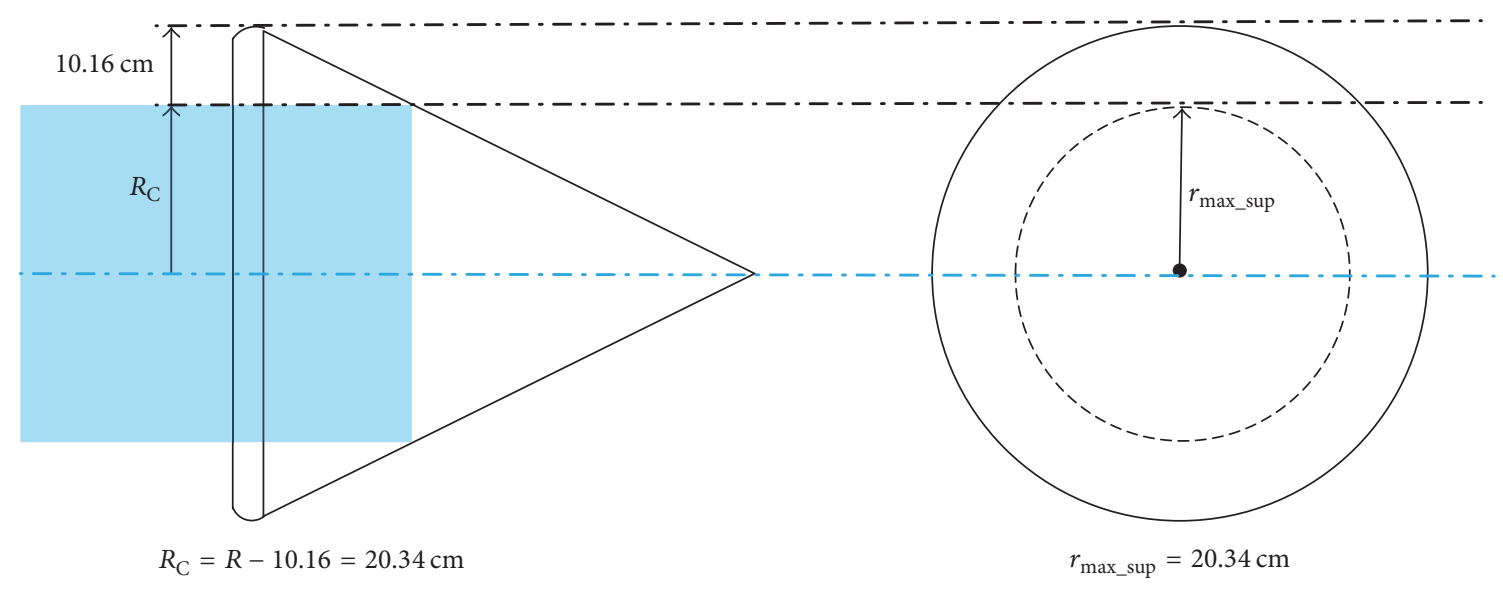

(a)

(b)

FIGURE 10: Visualized comparison between the flight test results of NASA AAR demonstration [21] and the theoretical analysis proposed in this paper for the probe-drogue docking success rate/probability. (a) Left view of the drogue and $R_{C}$ value, corresponding to a 90 percent probedrogue docking success rate in the flight test of NASA AAR demonstration [21]. (b) Main view of the drogue and $r_{\text {max_sup }}$ value, corresponding to larger than 89.44 percent probe-drogue docking success probability in the theoretical analysis proposed in this paper.

of the drogue is more than $90^{\circ}, P \_o p t i m a l$ will be 0 . Motivated by the above discussion, the pitch angle $\theta$ and yaw angle $\varphi$ of the drogue can be regarded as the penalty factor $c$ of $P$ initial. Thus, a concise and reasonable definition of penalty factor $c$ is given as follows:

$$
c(x)= \begin{cases}\frac{|| x|-\pi / 2|}{\pi / 2}, & |x| \in\left[0, \frac{\pi}{2}\right], \\ 0, & |x| \in\left(\frac{\pi}{2}, \pi\right]\end{cases}
$$

where $x \in[-\pi, \pi], x$ represents the deflection angle of the drogue, and $|x|$ represents the deflection amplitude of the drogue. When $|x|$ increases from $0 \rightarrow \pi / 2$, penalty factor $c(x)$ decreases from $1 \rightarrow 0$; when $|x| \in(\pi / 2, \pi], c(x)=0$.

Then, considering the relative attitude between the drogue and the probe, the P_optimal is defined as

$$
P_{\text {optimal }}=c(\theta) \cdot c(\varphi) \cdot P_{\text {initial }}= \begin{cases}\frac{|| \theta|-\pi / 2|}{\pi / 2} \cdot \frac{|| \varphi|-\pi / 2|}{\pi / 2} \cdot P_{\text {initial }}, & |\theta| \in\left[0, \frac{\pi}{2}\right] \text { and }|\varphi| \in\left[0, \frac{\pi}{2}\right] \\ 0, & \text { otherwise }\end{cases}
$$

where $\theta \in[-\pi, \pi]$ and $\varphi \in[-\pi, \pi]$. When $|\theta|$ and $|\varphi|$ increase from $0 \rightarrow \pi / 2$ and $c(\theta)$ and $c(\varphi)$ decrease from $1 \rightarrow 0$, P_optimal decreases from $P \_$initial $\rightarrow 0$; when $|\theta| \in(\pi / 2, \pi]$ or $|\varphi| \in(\pi / 2, \pi]$ and $c(\theta)=0$ or $c(\varphi)=0, P \_o p t i m a l=0$. We should note that, during the docking phase of aerial refueling, $30^{\circ}$ deflection of the drogue can have much influence on the probe-drogue docking.

\subsection{Considering the Accurate Measurements and UAV Control} for Docking Success Probability. Note that a successful docking for probe-drogue aerial refueling is not only dependent on the movement of the drogue center, but also related to accurate measurements for the parameters between probe and the drogue and the control of UAV. Firstly, the theory, experiment, and measurement precision of drogue location and attitude have been described in our previous work [6] in detail. So, in this study, we assume that accurate parameters of the drogue can be obtained precisely. Secondly, the actuation error described in this paper can be regarded as a token of the control of UAV. Namely, without actuation error can be regarded as the high accuracy of the control of UAV, while with actuation error can be regarded as the deviation of the control of UAV.

6.3. Considering the Statistical Probability Model for Docking Success Probability. Note that it can be meaningful and helpful to model the random factors in PDARS and give its statistical probability model using statistical methods based on the experimental data.

Currently, due to a lack of flight test data of UAV AAR for some practical reasons, the statistical probability model for probe-drogue docking success probability cannot be built for the time being but deserves further research in future. Nevertheless, in order to make the proposed model of probedrogue docking success probability more comprehensive and reasonable, the movements of the drogue (center) under 


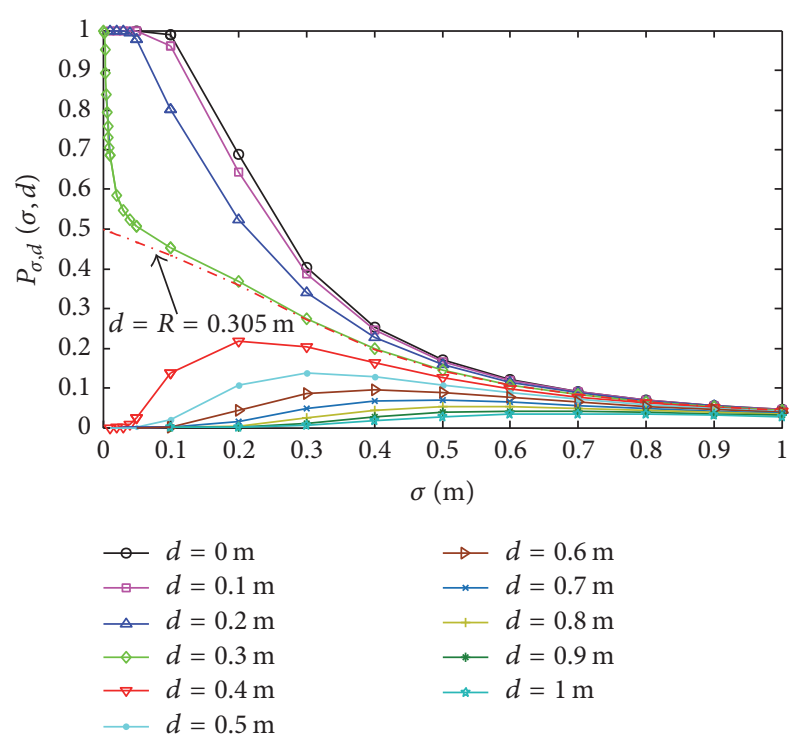

Figure 11: $P_{\sigma, d}(\sigma, d)$ versus $\sigma$ and $d$.

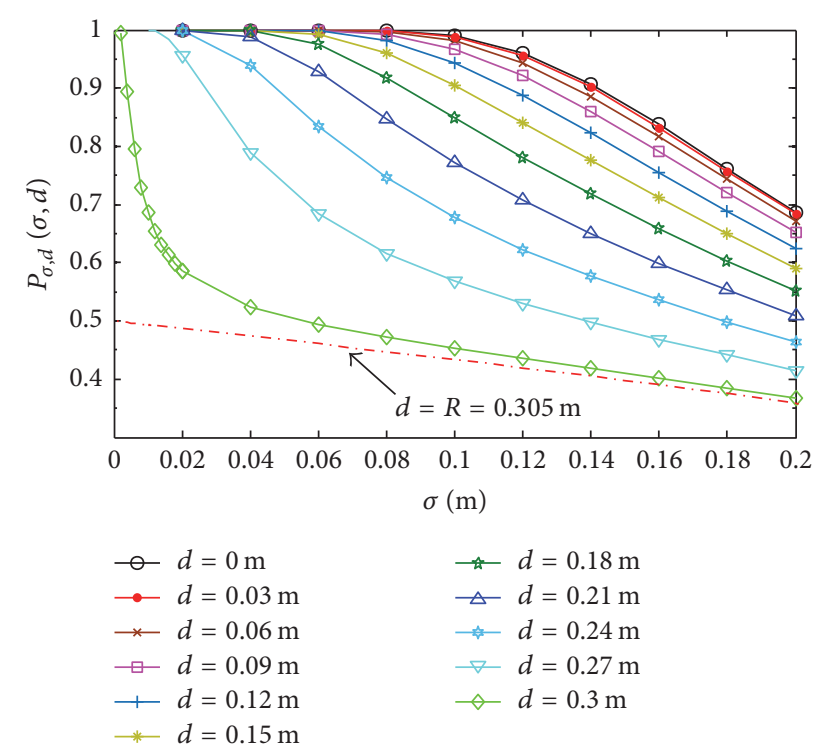

Figure 12: $P_{\sigma, d}(\sigma, d)$ versus $\sigma$ and $d$ (local detail).

disturbance, the actuation error (namely, the control of UAV), the relative attitude between the drogue and the probe, and the accurate measurements for the parameters between probe and the drogue are all taken into consideration. Note that the movements of the drogue (center), the actuation error (namely, the control of UAV), and the relative attitude between the drogue and the probe can be regarded as the intuitive reflection of the factors that affect probe-drogue docking success probability. Besides, a comparison between the theoretical analysis proposed in this paper and the flight test results of NASA AAR demonstration is reported. For the probe-drogue docking success probability, the flight test results of NASA AAR demonstration are consistent with the theoretical analysis proposed in this paper.
It is expected that, through an approach based on the mathematical model rather than human heuristic insight, UAV can obtain the probe-drogue docking success probability, which can be of some help for UAV AAR. Meanwhile, statistical probability model based on the flight test data of UAV AAR for probe-drogue docking success probability deserves further research and concern. And, whether artificial intelligence (such as deep learning) can be used to train the UAV to carry out aerial refueling also deserves concern and future study [28].

6.4. The Applicability of the Research. The research can provide a useful and valuable theoretical basis/reference, the probe-drogue docking success probability, for the practical engineering of UAV probe-drogue autonomous aerial refueling during the close docking stage. For example, during the close docking phase of UAV probe-drogue autonomous aerial refueling, (1) when the estimated probe-drogue docking success probability $P_{\text {estimated }} \geq$ Threshold, the UAV can execute the docking maneuver under safe conditions; (2) when the estimated probe-drogue docking success probability $P_{\text {estimated }}<$ Threshold, the docking maneuver can be regarded as unsafe, and the docking maneuver should be terminated. Without loss of generality, the Threshold can be set as 0.9. Note that the value of the Threshold should be determined by practical engineering.

\section{Conclusions}

(1) This paper numerically simulates the docking success probability during the docking phase of PDARS. Considering the facts that the receiver aircraft operates docking maneuver when the probe is in front of the drogue, and the atmospheric disturbance affects the movement of the drogue, this paper provides the theoretical estimation of probe-drogue docking success probability before docking maneuver when the probe is in front of the equilibrium position of drogue center.

(2) Reasonable assumptions for the distribution of the drogue center position under atmospheric disturbance are provided, according to the prior information during the docking phase of PDARS.

(3) A model of probe-drogue docking success probability is established, by converting the probe-drogue docking success probability to the probability of the drogue center located in a specific area.

(4) The simulation results can provide a theoretical basis for docking maneuver decision during the docking phase of PDARS. It is expected that, through an approach based on the mathematical model rather than human heuristic insight, UAV can obtain the probe-drogue docking success probability, which can be of some help for UAV AAR.

(5) The future work will include the following: (a) a more comprehensive model to estimate the docking success probability is currently under consideration; 


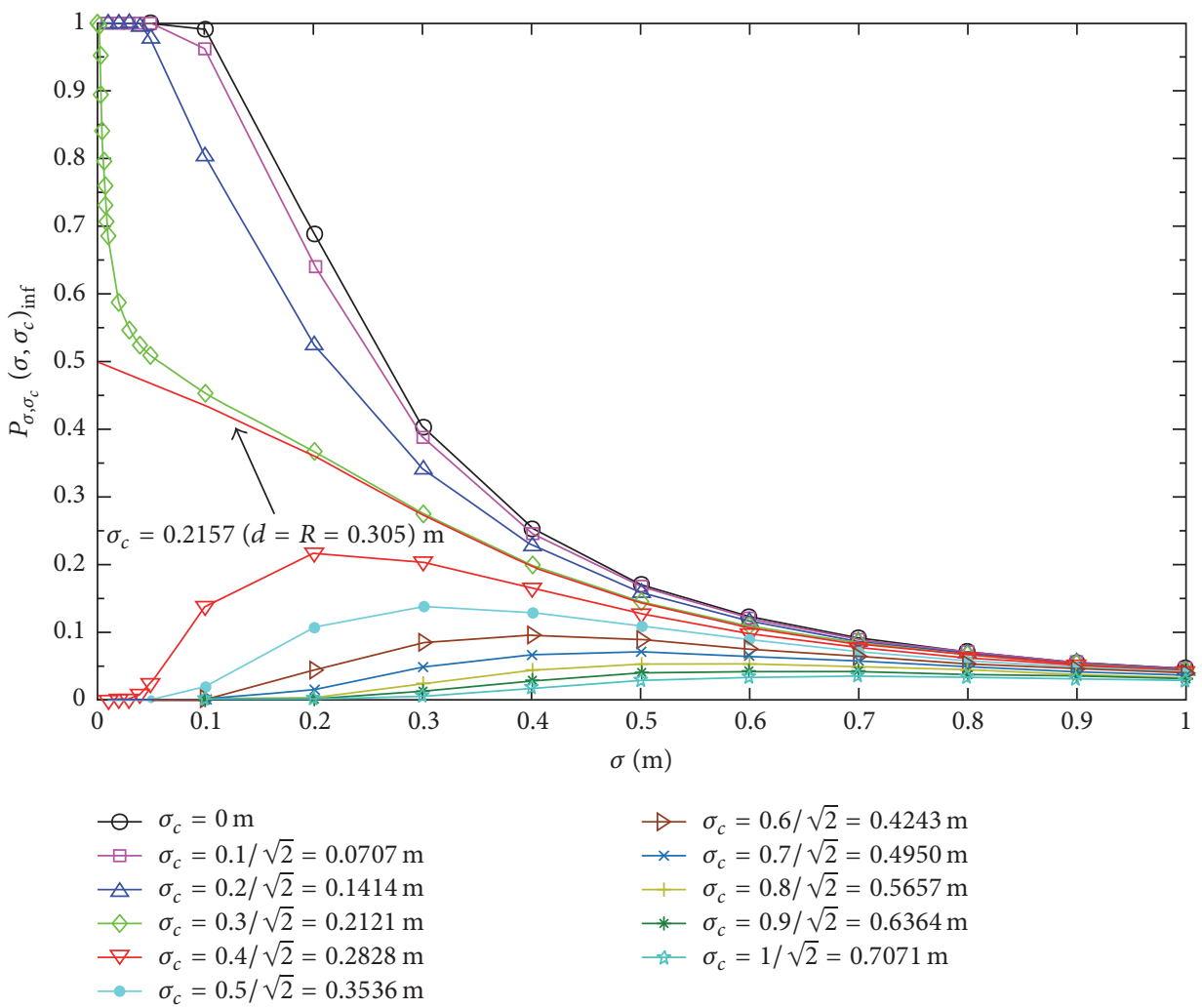

Figure 13: $P_{\sigma, \sigma_{c}}\left(\sigma, \sigma_{c}\right)_{\text {inf }}$ versus $\sigma$ and $\sigma_{c}$.

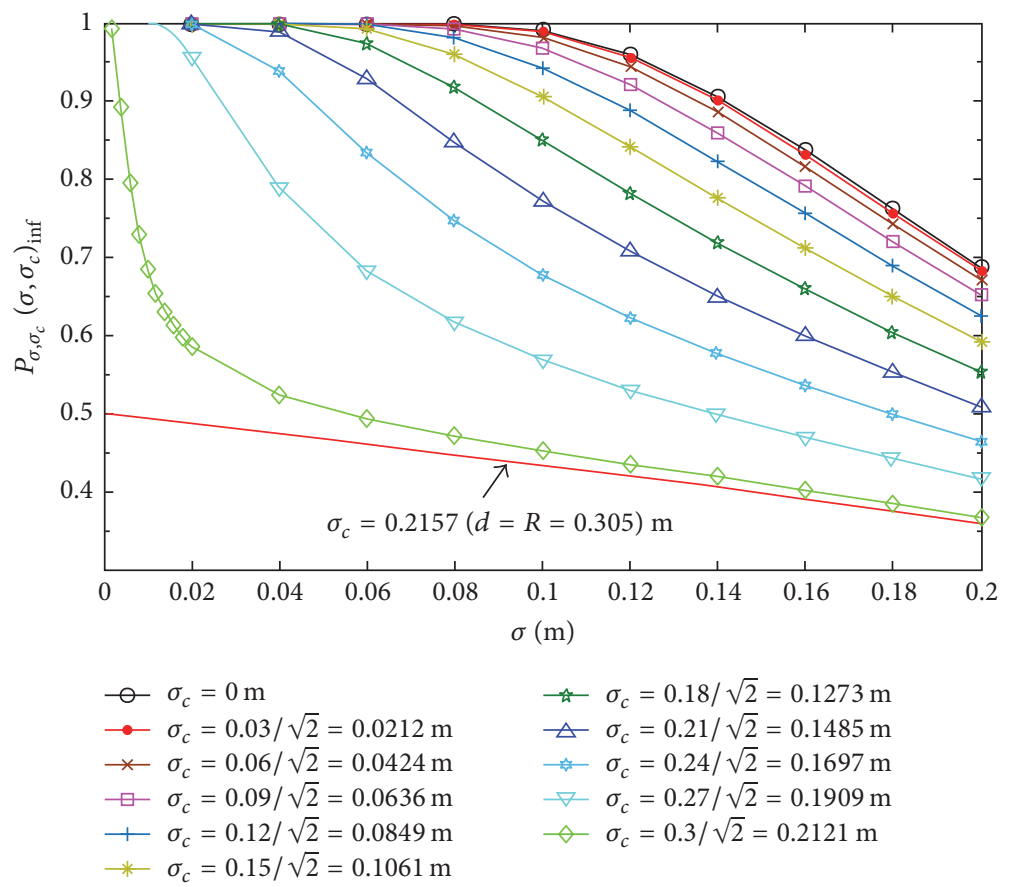

Figure 14: $P_{\sigma, \sigma_{c}}\left(\sigma, \sigma_{c}\right)_{\text {inf }}$ versus $\sigma$ and $\sigma_{c}$ (local detail). 
(b) to further demonstrate the correctness of the proposed model, the simulated probability results will be compared with real data in future research; (c) the proposed approach needs formal verification and validation by the flight test of AAR.

\section{Conflicts of Interest}

The authors declare that there are no conflicts of interest regarding the publication of this paper.

\section{Acknowledgments}

This work was cosupported by the National Basic Research Program of China (nos. 2012CB316301 and 2013CB329403) and the National Natural Science Foundation of China (nos. 61473307, 61304120, 61603411, 61273023, and 61332007).

\section{References}

[1] J. P. Nalepka and J. L. Hinchman, "Automated aerial refueling: extending the effectiveness of UAVs," in Proceedings of the AIAA Modeling and Simulation Technologies Conference and Exhibit, pp. 2005-6005, San Francisco, Calif, USA.

[2] X. M. Dong, Y. J. Xu, and B. Chen, "Progress and challenges in automatic aerial refueling," Journal of Air Force Engineering University: Natural Science Edition, vol. 9, no. 6, pp. 1-5, 2008.

[3] C. D. Mu and B. R. Li, "Vision-based autonomous aerial refueling," Journal of Tsinghua University (Science and Technology), vol. 52, no. 5, pp. 670-676, 2012.

[4] P. R. Thomas, U. Bhandari, S. Bullock, T. S. Richardson, and J. L. Du Bois, "Advances in air to air refuelling," Progress in Aerospace Sciences, vol. 71, pp. 14-35, 2014.

[5] Q. Quan, Z. B. Wei, J. Gao, R. F. Zhang, and K. Y. Cai, "A survey on modeling and control problems for probe and drogue autonomous aerial refueling at docking stage," Acta Aeronautica et Astronautica Sinica, vol. 35, no. 9, pp. 2390-2410, 2014.

[6] X. F. Wang, X. W. Kong, J. H. Zhi, Y. Chen, and X. M. Dong, "Real-time drogue recognition and 3D locating for UAV autonomous aerial refueling based on monocular machine vision," Chinese Journal of Aeronautics, vol. 28, no. 6, pp. 16671675, 2015.

[7] L. Pollini, G. Campa, F. Giulietti, and M. Innocenti, "Virtual simulation set-up for UAVs aerial refuelling," in Proceedings of the AIAA Modeling and Simulation Technologies Conference and Exhibit, pp. 2003-5682, Austin, Texas, USA, August 2003.

[8] R. Mati, L. Pollini, A. Lunghi, M. Innocenti, and G. Campa, "Vision-based autonomous probe and drogue aerial refueling," in Proceedings of the 14th Mediterranean Conference on Control and Automation, (MED '06), Ancona, Italy, June 2006.

[9] H. W. Xie and H. L. Wang, "Binocular vision-based short-range navigation method for autonomous aerial refueling," Journal of Beijing University of Aeronautics and Astronautics, vol. 37, no. 2, pp. 206-209, 2011.

[10] C.-I. Chen and R. Stettner, "Drogue tracking using 3D flash lidar for autonomous aerial refueling," in Proceedings of the SPIE 8037, Laser Radar Technology and Applications XVI, 80370Q, Orlando, Florida, USA, June 2008.

[11] M. Q. Hu, X. Nie, and L. M. Wang, "Determination of hose static catenary shape in probe-drogue in-flight refueling system,"
Journal of Air Force Engineering University: Natural Science Edition, vol. 10, no. 5, pp. 22-26, 2009.

[12] M. Q. Hu, P. Liu, X. Nie, and R. X. Zhou, "Influence of air turbulence on the movement of hose-drogue," Flight Dynamics, vol. 28, no. 5, pp. 20-23, 2010.

[13] L. Y. Zhang, H. Zhang, Y. Yang, and L. Huang, "Dynamics modeling and simulation of docking process in aerial refueling," Acta Aeronautica et Astronautica Sinica, vol. 33, no. 7, pp. 13471354, 2012.

[14] K. Ro and J. W. Kamman, "Modeling and simulation of hose-paradrogue aerial refueling systems," Journal of Guidance, Control, and Dynamics, vol. 33, no. 1, pp. 53-63, 2010.

[15] H. T. Wang, X. M. Dong, H. F. Dou, and J. P. Xue, "Dynamic modeling and characteristics analysis of hose-paradrogue aerial refueling system," Journal of Beijing University of Aeronautics and Astronautics, vol. 40, no. 1, pp. 92-98, 2014.

[16] J. Zhang, S. Z. Yuan, and Q. Q. Gong, "Modeling and control of shaking motion of aerial refueling hose-drogue," Journal of System Simulation, vol. 28, no. 2, pp. 388-395, 2016.

[17] H. T. Wang, X. M. Dong, J. P. Xue, and J. L. Liu, "Dynamic modeling of a hose-drogue aerial refueling system and integral sliding mode backstepping control for the hose whipping phenomenon," Chinese Journal of Aeronautics, vol. 27, no. 4, pp. 930-946, 2014.

[18] H. T. Wang, X. M. Dong, J. Guo, J. L. Liu, and J. Wang, “Dynamics modeling and analysis of hose whipping phenomenon of aerial refueling hose-drogue assembly," Acta Aeronautica et Astronautica Sinica, vol. 36, no. 9, pp. 3116-3127, 2015.

[19] H. L. Wang, Y. Du, and W. D. Gai, "Precise docking control in unmanned aircraft vehicle automated aerial refueling," Journal of Beijing University of Aeronautics and Astronautics, vol. 37, no. 2, pp. 206-209, 2011.

[20] M. D. Tandale, R. Bowers, and J. Valasek, "Trajectory tracking controller for vision-based probe and drogue autonomous aerial refueling," Journal of Guidance, Control, and Dynamics, vol. 29, no. 4, pp. 846-857, 2006.

[21] R. P. Dibley, M. J. Allen, and N. Nabaa, "Autonomous airborne refueling demonstration phase I flight-test results," in Proceedings of the AIAA Atmospheric Flight Mechanics Conference, pp. 1131-1149, Reston, Virginia, USA, August 2007.

[22] J. L. Hansen, J. E. Murray, and N. V. Campos, "The NASA Dryden AAR project: a flight test approach to an aerial refueling system," in Proceedings of the Collection of Technical Papers AIAA Atmospheric Flight Mechanics Conference, pp. 477-495, Reston, Virginia, USA, August 2004.

[23] M. J. Vachon, R. J. Ray, and C. Calianno, "Calculated drag of an aerial refueling assembly through airplane performance analysis," in Proceedings of the 42nd AIAA Aerospace Sciences Meeting and Exhibit, Reno, Nevada.

[24] A. Dogan, W. Blake, and C. Haag, "Bow wave effect in aerial refueling: computational analysis and modeling," Journal of Aircraft, vol. 50, no. 6, pp. 1856-1868, 2013.

[25] U. Bhandari, P. R. Thomas, S. Bullock, T. S. Richardson, and J. L. du Bois, "Bow wave effect in probe and drogue aerial refuelling," in Proceedings of the AIAA Guidance, Navigation, and Control (GNC) Conference, Boston, MA, USA, August 2013.

[26] X. H. Dai, Z. B. Wei, and Q. Quan, "Modeling and simulation of bow wave effect in probe and drogue aerial refueling," Chinese Journal of Aeronautics, vol. 29, no. 2, pp. 448-461, 2016.

[27] Z. B. Wei, X. H. Dai, Q. Quan, and K. Y. Cai, "Drogue dynamic model under bow wave in probe-and-drogue refueling," IEEE 
Transactions on Aerospace and Electronic Systems, vol. 52, no. 4, pp. 1728-1742, 2016.

[28] X. F. Wang, X. M. Dong, X. W. Kong, J. M. Li, and B. Zhang, "Drogue detection for autonomous aerial refueling based on convolutional neural networks," Chinese Journal of Aeronautics, vol. 30, no. 1, pp. 380-390, 2017. 


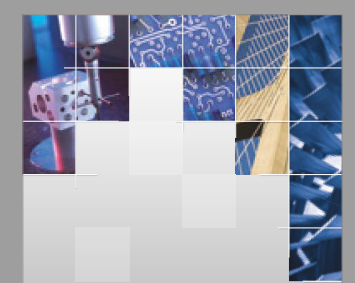

\section{Enfincering}
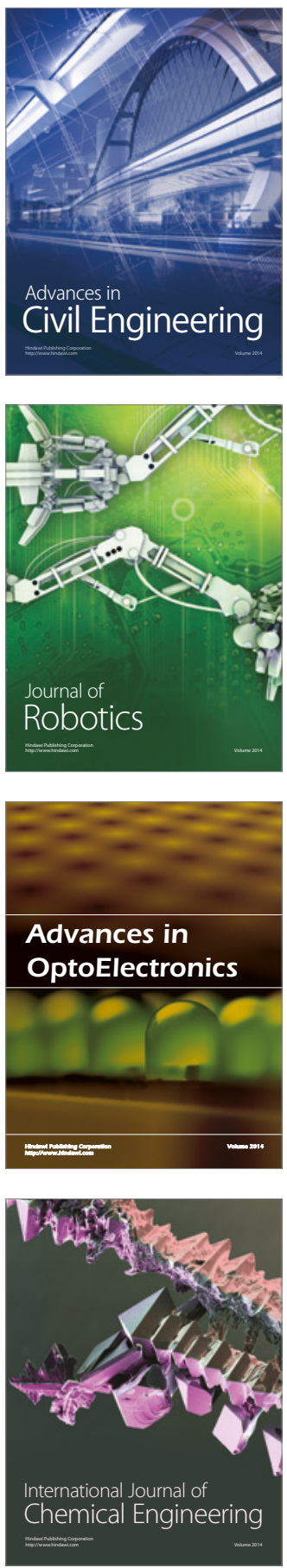

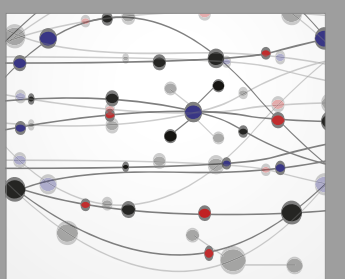

The Scientific World Journal

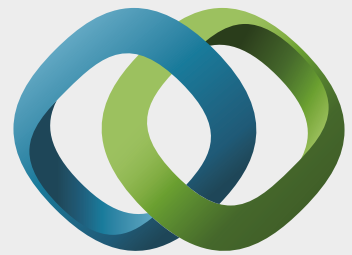

\section{Hindawi}

Submit your manuscripts at

https://www.hindawi.com
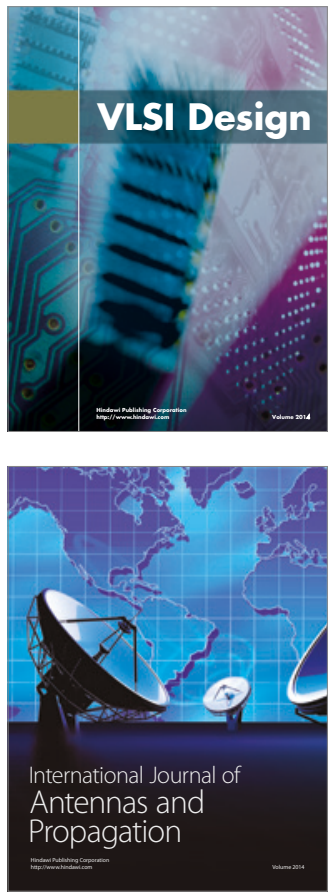

\section{Rotating}

Machinery
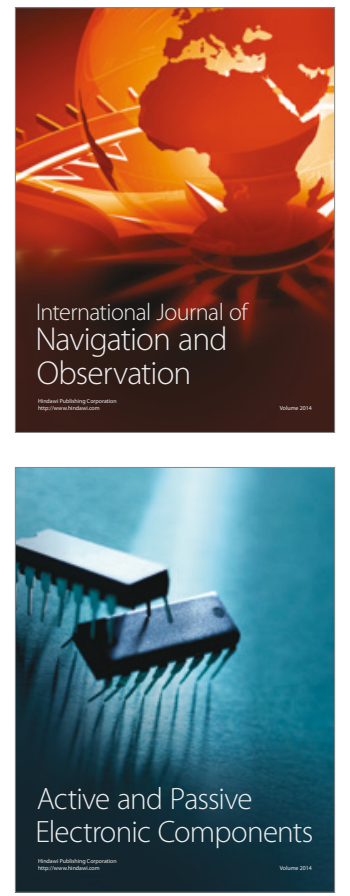
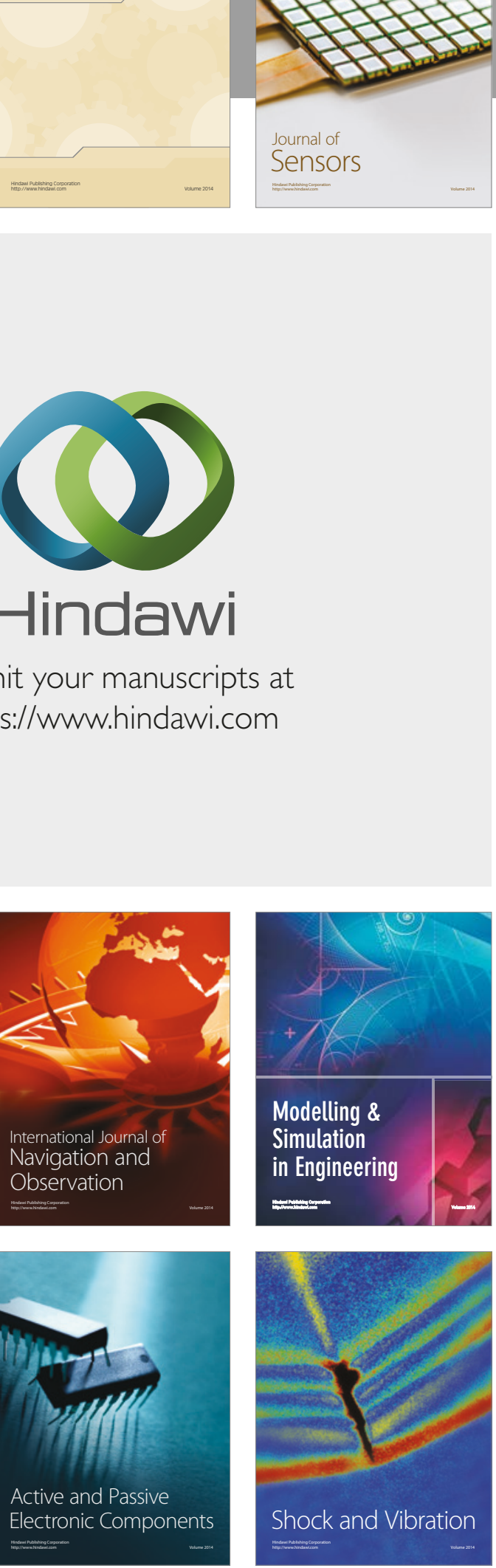
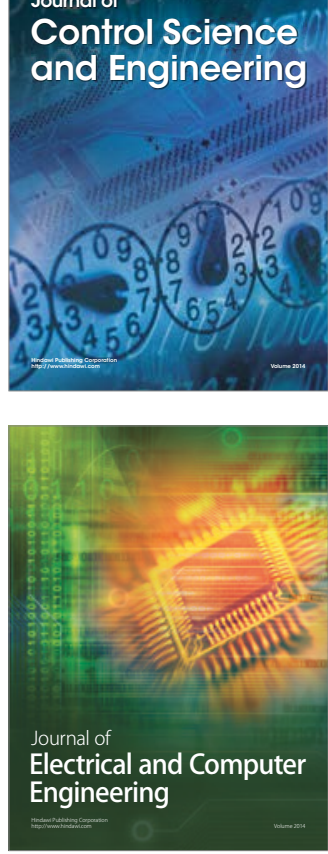

Distributed

Journal of

Control Science

and Engineering
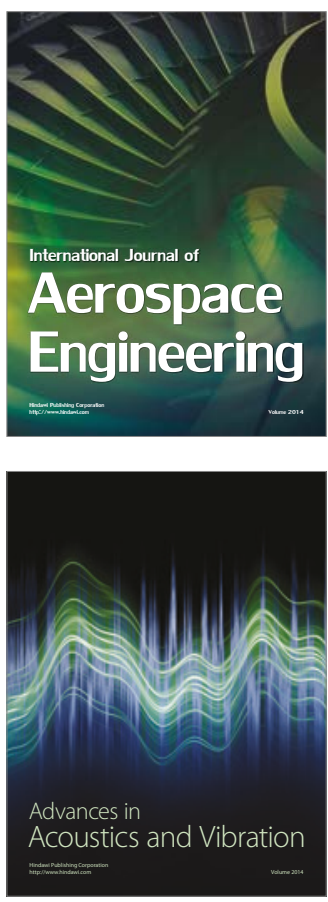

Sensor Networks 